Check for updates

Cite this: RSC Adv., 2019, 9, 36751

Received 22nd August 2019

Accepted 28th October 2019

DOI: 10.1039/c9ra06595d

rsc.li/rsc-advances

\section{Recyclable functionalized polymer films for the efficient removal of hexavalent chromium from aqueous solutions $\uparrow$}

\author{
Weikang Liu, ${ }^{a}$ Mei Wang, ${ }^{c}$ Zhigang Wen, ${ }^{c}$ Zhong Li, ${ }^{* a}$ Liang Yang (D) *b \\ and Changlong Jiang (DD $* b$
}

In this study, polyethylenimine-functionalized poly(vinyl alcohol) (PEI-PVA) films were prepared for the first time to remove aqueous $\mathrm{Cr}(\mathrm{VI})$. The results indicate that our PEI-PVA films have an excellent potential for $\mathrm{Cr}(\mathrm{vI})$ removal and their maximum removal capacity was $396.83 \mathrm{mg} \mathrm{g}^{-1}$. The optimized $\mathrm{pH}$ value was 2 , the adsorption of $\mathrm{Cr}(\mathrm{VI})$ was fitted to the Langmuir model, and the kinetics of uptake could be described well by a pseudo-second-order rate model. Taking into account the simplified separation method of adsorbents and solutions, we used a PVA film as a carrier in which PEI-PVA microspheres were filled to obtain a PEI-PVA functionalized film (PPF). The PPF shows a great efficiency in the removal of $\mathrm{Cr}(\mathrm{VI})$ ions in solution, which can absorb and reduce the $\mathrm{Cr}(\mathrm{VI})$ ion concentration in the solution in 90 min. PPF has excellent selectivity and the removal efficiency of $\mathrm{Cr}(\mathrm{vI})$ ions in the presence of co-existing ions is not reduced. It also has good recycling properties; the removal efficiency remains at $77 \%$ over four cycles. The removal mechanism of $\mathrm{Cr}(\mathrm{VI})$ ions by PEI-PVA microspheres involves the reduction of the adsorbed $\mathrm{Cr}(\mathrm{VI})$ ions to $\mathrm{Cr}(\mathrm{III})$ ions, which are less toxic.

\section{Introduction}

The increasing water pollution by widespread heavy metals is of great concern due to their toxicity, inability to be biodegraded, and the wide range of health hazards they pose because they are likely to be accumulated in living organisms via the food chain, causing a long-term potential threat to human health and natural ecosystems. Hexavalent chromium $\mathrm{Cr}(\mathrm{vI})$, one of the most toxic contaminants, is released in the environment due to various anthropogenic activities and poses a serious threat to human beings as well as flora and fauna., ${ }^{1,2}$ The main causes of anthropogenic chromium pollution in groundwater are metal electroplating, steelworks manufacturing, leather tanning and several others. ${ }^{3}$ It is found that chromium exists in the environment in two stable states: trivalent $\mathrm{Cr}(\mathrm{III})$ and hexavalent $\mathrm{Cr}(\mathrm{vI})$, and the toxicity of the hexavalent form is five hundred times greater than that of the trivalent form. ${ }^{4-6} \mathrm{Cr}^{3+}$ is an essential element for humans and is much less toxic. $\mathrm{Cr}^{6+}$ is one of the extremely toxic heavy metals to biological systems due to

${ }^{a}$ School of Chemical Engineering, Anhui University of Science and Technology, Huainan, Anhui, 232001, China. E-mail: zhongli@aust.edu.cn

${ }^{b}$ Institute of Intelligent Machines, Chinese Academy of Sciences, Hefei, Anhui, 230031, China.E-mail: yangliang@iim.ac.cn

${ }^{c}$ The State Centre of Quality Surpervision and Inspection for Camellia Products, Ganzhou, Jiangxi, 341000, China

$\dagger$ Electronic supplementary information (ESI) available. See DOI: 10.1039/c9ra06595d its carcinogenic and corrosive properties, and can cause many health problems such as liver damage, pulmonary congestion and vomiting. ${ }^{7-10}$ Hence, various agencies such as the WHO and the US EPA have set a tolerable limit of $0.05 \mathrm{mg} \mathrm{L}^{-1}$ for dissolved $\mathrm{Cr}(\mathrm{vI})$ in drinking water, and that for all forms of chromium is $2 \mathrm{mg} \mathrm{L}^{-1}$. $^{\mathbf{1 , 6}}$ Therefore, it is very important to remove $\mathrm{Cr}(\mathrm{vI})$ from wastewater or reduce $\mathrm{Cr}(\mathrm{VI})$ to $\mathrm{Cr}(\mathrm{III})$ prior to discharge into the environment. Until now, many methods have been used to treat Cr-contaminated solutions, such as coprecipitation, ${ }^{\mathbf{1 1}}$ membrane filtration, ${ }^{\mathbf{1 2}}$ ion exchange, ${ }^{\mathbf{1 3}}$ and adsorption. ${ }^{\mathbf{1 4}}$ Among these, adsorption is preferred because of its simple design and environmental friendliness.

Adsorption is a process in which pollutants are adsorbed on the surface of an adsorbent. Until now, different types of adsorbent materials have been widely studied for the removal of $\mathrm{Cr}$ (vi) from aqueous solutions including activated carbon, clay minerals, organic resins and some nanomaterials. ${ }^{15}$ However, conventional adsorbents or nanomaterials often show a limited adsorption capacity or cannot be easily separated or even cause potential secondary pollution because they do not have enough surface area, functional groups, hydrophilic surfaces or recycling methods. After adsorption, the removal of nanoparticles is a bit of a tedious job due to their small size, which can cause health and environmental issues. ${ }^{16}$ Basically, the separation of nanoparticles from aqueous solutions depends on their nature. Many different methods have been attempted for separating nanoparticles from aqueous solutions and the most important include magnetism, cross-flow filtration, and centrifugation. ${ }^{17}$ 
Generally, magnetic nanoparticles contain magnetic elements such as iron, nickel, and cobalt. ${ }^{18}$ These types of nanoparticles can be separated from water easily by high gradient magnetic separation. In fact, these magnetic nanoparticles do not have the ability to remove the contaminants. In fact, these magnetic nanoparticles do not have the ability to remove the contaminants, they just play a supporting role in separating adsorbents and this separation method increases the use-cost of adsorbents. Therefore, there is a strong need to develop a cheap and environmentally friendly solid adsorbent with high efficiency for $\mathrm{Cr}(\mathrm{vI})$ removal and easy separation from solutions.

Poly(vinyl alcohol) (PVA) is a water-soluble synthetic polymer containing many reactive hydroxyl groups. Due to its characteristics of low cost, non-toxicity, excellent biocompatibility, good mechanical strength and chemical stability, PVA has been extensively used in biomedical and pharmaceutical applications. ${ }^{19}$ Herein, we used PVA to prepare microspheres, which were modified with PEI in a subsequent reaction. These PEI-PVA microspheres exhibited excellent efficiency for the removal of hexavalent chromium ions from aqueous solutions. For simplifying the separation of adsorbents and solutions, we used a PVA film as a carrier and filled PEI-PVA microspheres into the PVA functional film (PPF). Subsequent tests showed that this functionalized film has a high removal efficiency for $\mathrm{Cr}(\mathrm{vI})$ ions, and has good reusability and easy separation characteristics.

\section{Experimental}

\subsection{Materials}

Natural graphite flakes were supplied by Sigma Chemical Corporation. Vinyl acetate (VAc) and divinylbenzene (DVB) were purchased from Alfa Aesar. PEI (MW 1800) was obtained from Sigma-Aldrich. PVA (MW 1000), benzoyl peroxide (BPO), $\mathrm{NaCl}$, methanol, $\mathrm{NaOH}, \mathrm{N}, \mathrm{N}$-dimethylformamide (DMF) and epichlorohydrin $(\mathrm{ECH})$ were of analytical grade and were purchased from Shanghai Chemical Reagent Corporation. Aqueous solutions were all prepared using ultrapure water $(18.2 \mathrm{M} \Omega \mathrm{cm})$ from a Millipore water purification system. All chemicals were used in the experiments directly without any further purification.

\subsection{Fabrication of PVA microspheres}

PVAc microspheres were prepared by the method described in the previous literature. In a typical experiment, $0.8 \mathrm{~g}$ of BPO (as the initiator) was dispersed in a mixture of $19 \mathrm{~mL}$ of VAc and $1 \mathrm{~mL}$ of DVB (as the cross-linker) under stirring. The mixture was added into a solution of $200 \mathrm{~mL}$ of water, $4 \mathrm{~g}$ of PVA and $6 \mathrm{~g}$ of $\mathrm{NaCl}$ in a $500 \mathrm{~mL}$ flask equipped with a magnetic stirrer. The polymerization system was then heated to $60{ }^{\circ} \mathrm{C}$ and maintained at $60{ }^{\circ} \mathrm{C}$ for $2 \mathrm{~h}$, and finally, the temperature was increased to $70{ }^{\circ} \mathrm{C}$ and kept for $2 \mathrm{~h}$. The reaction mixture was cooled to room temperature and thoroughly washed with deionized water and ethanol to remove excess VAc and DVB.

The PVA microspheres were obtained by the methanolysis of PVAc microspheres. $0.25 \mathrm{~g}$ of PVAc microspheres were dispersed into a mixture of $50 \mathrm{~mL}$ of methanol, $1.5 \mathrm{~g}$ of $\mathrm{NaOH}$ and $5 \mathrm{~mL}$ of deionized water. The above solution was stirred gently at $50{ }^{\circ} \mathrm{C}$ for $12 \mathrm{~h}$. Next, the resulting microspheres were collected and washed several times with deionized water and ethanol.

\subsection{Functionalization of PVA microspheres}

$0.15 \mathrm{~g}$ PVA microspheres were dispersed into a mixture of DMF $(25 \mathrm{~mL})$ and $\mathrm{ECH}(25 \mathrm{~mL})$. The above mixture was stirred at $45{ }^{\circ} \mathrm{C}$ for $12 \mathrm{~h}$. Next, the resulting microspheres were washed several times with deionized water and ethanol and added to a solution of $0.5 \mathrm{~g}$ PEI in $25 \mathrm{~mL}$ deionized water and stirred at $80{ }^{\circ} \mathrm{C}$ for another $12 \mathrm{~h}$. The resulting PEI-PVA microspheres were collected and washed several times with ethanol and deionized water to remove the residual PEI.

\subsection{Preparation of functional microspheres-PVA film}

$0.1 \mathrm{~g}$ of PEI-PVA microspheres were dispersed in suitable GO and 5\% (w/v) PVA solution under ultrasonic conditions and then poured into a culture dish. As the water evaporated, the remaining PVA formed a thin, mechanically durable film on the top of the dish. The circumference of the PVA film on the dish was carved out, and the film was peeled off using tweezers.

\subsection{Removal of $\mathrm{Cr}(\mathrm{vI})$ from aqueous solutions}

The PEI-PVA microspheres (10 mg) were added to $20 \mathrm{~mL}$ of $\mathrm{Cr}(\mathrm{vI})$ aqueous solutions. After being shaken for $36 \mathrm{~h}$, the solid and liquid were separated by filtration using a $0.22 \mu \mathrm{m}$ microporous membrane filter. The influence of the initial $\mathrm{Cr}(\mathrm{vI})$ concentration, $\mathrm{pH}$, contact time and temperature on the removal efficiency of $\mathrm{Cr}(\mathrm{vI})$ were investigated. After that, the removal capacity $\left(q_{\mathrm{e}}\right)$ of $\mathrm{Cr}(\mathrm{vI})$ was calculated according to the following equation.

$$
q_{\mathrm{e}}=\frac{\left(C_{0}-C_{\mathrm{e}}\right) V}{m}
$$

where $C_{0}$ and $C_{\mathrm{e}}$ are the initial and equilibrium concentrations of $\mathrm{Cr}(\mathrm{vI})$ in the aqueous solution, respectively; $V(\mathrm{~L})$ is the volume of the solution and $m$ is the weight of the adsorbent used (g).

\subsection{Determination of $\operatorname{Cr}(\mathrm{vI})$ concentration}

The concentration of $\mathrm{Cr}(\mathrm{vI})$ was analyzed by the spectrophotometric method using 1,5-diphenylcarbohydrazide. In each $\mathrm{Cr}(\mathrm{vI})$ measurement experiment, a $0.2 \mathrm{~mL}$ solution containing $\mathrm{Cr}(\mathrm{vI})$ was transferred into a $50 \mathrm{~mL}$ Erlenmeyer flask. Approximately $50 \mathrm{~mL}$ of deionized water, $0.5 \mathrm{~mL}$ of $\mathrm{H}_{2} \mathrm{SO}_{4}$ solution $(50 \%)$, $0.5 \mathrm{~mL}$ of $\mathrm{H}_{3} \mathrm{PO}_{4}$ solution (50\%), and $2 \mathrm{~mL}$ of 1,5-diphenylcarbohydrazide solution were successively added to the $\mathrm{Cr}(\mathrm{vI})$ solution. The mixture was vigorously shaken for $1 \mathrm{~min}$ and collected in a centrifuge tube after being left to stand for $5 \mathrm{~min}$. The resultant sample solution was transferred to a spectrophotometer for analysis at $540 \mathrm{~nm}$.

\subsection{Characterization and instruments}

The morphology of the PEI-PVA microspheres was determined by scanning electron microscopy (SEM, Sirion 200, FEI Co., 
USA); Fourier transform infrared spectra of PVAc and PEI-PVA microspheres were recorded on a spectrophotometer (FT-IR, Nicolet 8700, Thermo Scientific Instrument Co., USA) in the wavenumber range of $4000-500 \mathrm{~cm}^{-1}$; the concentration of $\mathrm{Cr}(\mathrm{vI})$ was measured using a UV-vis spectrophotometer (UV 2550, Shimadzu Co., Japan) at a wavelength of $540 \mathrm{~nm}$; XPS measurements were also carried out (ESCALAB 250, Thermo-VG Scientific Co., USA).

\section{Results and discussion}

\subsection{Characterization of different materials}

The process of preparing PEI-functionalized PVA microspheres and filling them into the PVA film is shown in Scheme 1. We first prepared the PVA microspheres and then introduced PEI on the surface of the microspheres through a series of chemical reactions. As shown in Fig. 1A, the microspheres had a spherical form and were monodispersed. The PEI-PVA microspheres had a narrow size distribution ranging from $1.6 \mu \mathrm{m}$ to $2.8 \mu \mathrm{m}$, and the average microsphere diameter was approximately $2.05 \mu \mathrm{m}$ (Fig. 1B). After the PEI-PVA microsphere particles were mixed with the $5 \%(\mathrm{w} / \mathrm{v})$ PVA solution, the mixed solution was poured into a culture dish. As a safe, nontoxic, water-soluble polymer, PVA is often used as a film former. As the water evaporated, the remaining PVA formed a thin, transparent, mechanically durable film on top of the dish. As shown in Fig. 1C, we can see that the PEI-PVA microspheres scattered on the surface of the film, and Fig. 1D shows that the thickness of the film is about 60 $\mu \mathrm{m}$.

The synthetic route for the preparation of PEI-PVA microspheres is shown in Scheme 1. In order to prove that PEI polymers were introduced successfully, FT-IR measurements were carried out. As shown in the spectrum of PVA (Fig. S1 $\dagger$ ), the broad band at $3422 \mathrm{~cm}^{-1}$ can be assigned to the $\mathrm{O}-\mathrm{H}$ stretching vibration and the band at $2923 \mathrm{~cm}^{-1}$ can be attributed to the $\mathrm{C}-\mathrm{H}$ stretching of the ethyl group, while the strong band at $1718 \mathrm{~cm}^{-1}$ corresponds to $\mathrm{C}=\mathrm{O}$ vibrations; ${ }^{20}$ these characteristic peaks indicate that PVA contained abundant oxygencontaining groups, which made it easier to introduce PEI polymers. After the modification of PVA by PEI, the IR spectrum of PEI-PVA microspheres exhibits obvious changes. In contrast, in the IR spectrum of PVA-PEI, the absorbance peaks of $\mathrm{C}-\mathrm{H}$ and $\mathrm{C}=\mathrm{O}$ bonds decreased to a large degree, indicating that

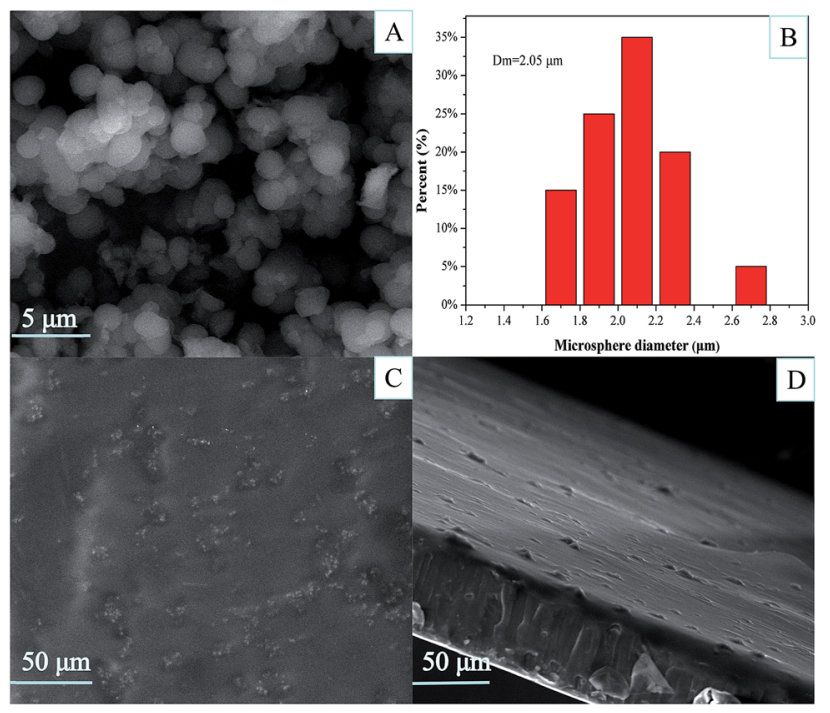

Fig. 1 SEM image (A) and size distribution (B) of the PEI-PVA microspheres; surface morphology (C) and thickness (D) of PEI-PVA functional film.

PVA was obtained through the methanolysis of PVAc. ${ }^{21}$ New bands appeared at 1308 and $1555 \mathrm{~cm}^{-1}$, which correspond to $\mathrm{N}-$ $\mathrm{H}$ bending vibrations, and a new band at $1635 \mathrm{~cm}^{-1}(-\mathrm{C}(=\mathrm{O})-$ $\mathrm{N}(-\mathrm{H})-)$ for PEI also appeared $\left(1635 \mathrm{~cm}^{-1}\right.$ for the $\mathrm{C}=\mathrm{O}$ stretching vibration in -NHCO-). ${ }^{22}$ This implied that PEI was successfully grafted on the surface of PVA microspheres. Branched polyethylenimine (PEI) has plenty of primary, secondary and tertiary amines on the macromolecular chains, which are considered as effective functional reagents to reduce $\mathrm{Cr}(\mathrm{VI})$ to $\mathrm{Cr}(\mathrm{III})$, which will be proved in the later XPS spectra.

\subsection{Effect of $\mathrm{pH}$ on adsorption}

The determination of the optimal $\mathrm{pH}$ value is very important in the adsorption process, since the $\mathrm{pH}$ affects not only the conversion of chromium species, but also the surface charge and protonation degree of functional groups on the active sites of the adsorbent. $\mathrm{Cr}(\mathrm{vI})$ can exist in aqueous solutions as various species, which is entirely dependent on the $\mathrm{pH}$ of the solution and its concentration. Fig. $\mathrm{S} 2 \mathrm{~B} \uparrow$ depicts the relative distribution of $\mathrm{Cr}(\mathrm{vI})$ species in aqueous solutions as a function of $\mathrm{pH}$. It

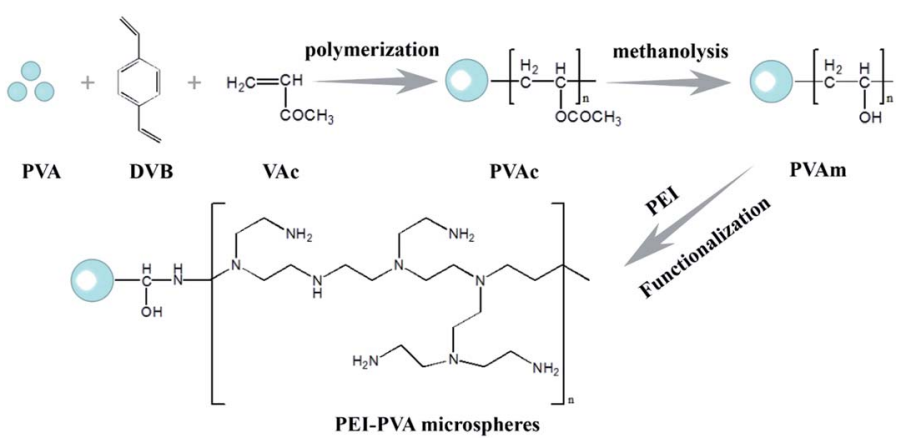

Scheme 1 Schematic illustration of the formation of PEI-PVA microspheres. 
shows that chromic acid $\left(\mathrm{H}_{2} \mathrm{CrO}_{4}\right)$ is predominant when the $\mathrm{pH}$ is less than 1 , the hydrogen chromate ion $\left(\mathrm{HCrO}_{4}{ }^{-}\right)$is predominant at $\mathrm{pH}$ values ranging from acidic $\mathrm{pH} 1$ to neutral $\mathrm{pH} 7$, whereas, above neutral $\mathrm{pH}$, only chromate ions $\left(\mathrm{CrO}_{4}{ }^{2-}\right)$ exist in the solution. Dichromate ions $\left(\mathrm{Cr}_{2} \mathrm{O}_{7}{ }^{2-}\right)$ occur when $\mathrm{Cr}(\mathrm{vI})$ concentration goes beyond $1 \mathrm{~g} \mathrm{~L}^{-1} \cdot{ }^{23}$ In this study, the effect of $\mathrm{pH}$ on $\mathrm{Cr}(\mathrm{vI})$ adsorption was tested at different $\mathrm{pH}$ values ranging from 2 to 12 . The maximum adsorption capacity was achieved at $\mathrm{pH} 2$, which was consistent with the previous study (Fig. S2A $\dagger$ ). The decrease in the adsorption capacity as the $\mathrm{pH}$ increased from 2 to 12 was attributed to the increase in the protonation of the amino groups on the PEI-PVA microspheres. The adsorption capacity was highly $\mathrm{pH}$ dependent, which indicates that electrostatic interaction and ion exchange play important roles in the adsorption of $\mathrm{Cr}(\mathrm{vI})$. Since the amino groups were easily protonated under acidic conditions, the surface charge of the adsorbent became positive, and the protonation degree of the amino groups increased as the $\mathrm{pH}$ decreased. These conditions were favorable for $\mathrm{Cr}(\mathrm{vI})$ adsorption onto the adsorbent by electrostatic interaction and ion exchange.

\subsection{Adsorption isotherms}

Adsorption isotherms are critical in optimizing the use of adsorbents, since they can not only be used to assess the adsorption capacity of the adsorbent, but also to describe how the adsorbate interacts with the adsorbent. Adsorption isotherm consists of two important models, namely, Langmuir and Freundlich isotherms. ${ }^{24}$ These two models are the most common isotherms used to describe the solid-liquid adsorption system. The Langmuir model is a single component (monolayer) adsorption model, which relates to the adsorption process at a particular homogeneous binding site on the adsorbent and comes to an end when the entire binding site is occupied. ${ }^{25}$ The linear form of the Langmuir adsorption isotherm model is presented as follows:

$$
\frac{C_{\mathrm{e}}}{q_{\mathrm{e}}}=\frac{1}{b q_{\mathrm{m}}}+\frac{C_{\mathrm{e}}}{q_{\mathrm{m}}}
$$

where $C_{\mathrm{e}}\left(\mathrm{mg} \mathrm{L}^{-1}\right)$ and $q_{\mathrm{e}}\left(\mathrm{mg} \mathrm{g}^{-1}\right)$ are $\mathrm{Cr}(\mathrm{vI})$ concentration and adsorption capacity at equilibrium, respectively; $q_{\mathrm{m}}\left(\mathrm{mg} \mathrm{g}^{-1}\right)$ is the maximum adsorption capacity, and $K\left(\mathrm{~L} \mathrm{mg}^{-1}\right)$ is the equilibrium constant related to adsorption energy. The Freundlich isotherm model is based on the adsorption of metal ions on heterogeneous binding sites, which may or may not be equivalent. ${ }^{26}$ The linear form of the Freundlich adsorption isotherm model is expressed as follows:

$$
\ln q_{\mathrm{e}}=\ln K_{\mathrm{f}}+\frac{1}{n} \ln C_{\mathrm{e}}
$$

where $K_{\mathrm{f}}$ and $n$ are related to the capacity and intensity of the adsorption, respectively.

By fitting our experimental data with the Langmuir and Freundlich adsorption isotherm models, the adsorption isotherm data fit the Langmuir model $\left(R^{2}=0.999\right.$, Fig. S $\left.3 \dagger\right)$ better than the Freundlich model. Based on the assumptions of the Langmuir model, the adsorption process proceeded by monolayer adsorption. The values of these parameters, as analyzed from the plots shown in Fig. S3, $\dagger$ are summarized in Table S1. $\dagger$ From the values of slope and intercept, the value of $q_{\mathrm{m}}$ was calculated to be $396.825 \mathrm{mg} \mathrm{g}^{-1}$. Compared to the reported adsorbents (Table S2 $\dagger$ ), ${ }^{27-36}$ PEI-PVA microspheres have a superb removal capacity toward $\mathrm{Cr}(\mathrm{VI})$. Therefore, PEI-PVA microspheres are a potential material for Cr-contaminated wastewater clean-up.

\subsection{Adsorption kinetics}

Adsorption kinetics, i.e., determining the pseudo-first or pseudo-second order kinetics, is another crucial parameter to examine the mechanism of the adsorption process in order to analyze the $\mathrm{Cr}(\mathrm{vI})$ uptake rate. In pseudo-first order kinetics, the rate of adsorption is proportional to the number of free binding sites on the adsorbent, whereas in pseudo-second order kinetics, it is directly proportional to the number of unoccupied binding sites. These two kinetic models are expressed as follows, respectively:

$$
\log \left(q_{\mathrm{e}}-q_{t}\right)=\log q_{\mathrm{e}}-\left(\frac{K_{1}}{2.303}\right) t
$$

$$
\frac{t}{q_{t}}=\frac{1}{K_{2} q_{\mathrm{e}}^{2}}+\left(\frac{1}{q_{\mathrm{e}}}\right) t
$$
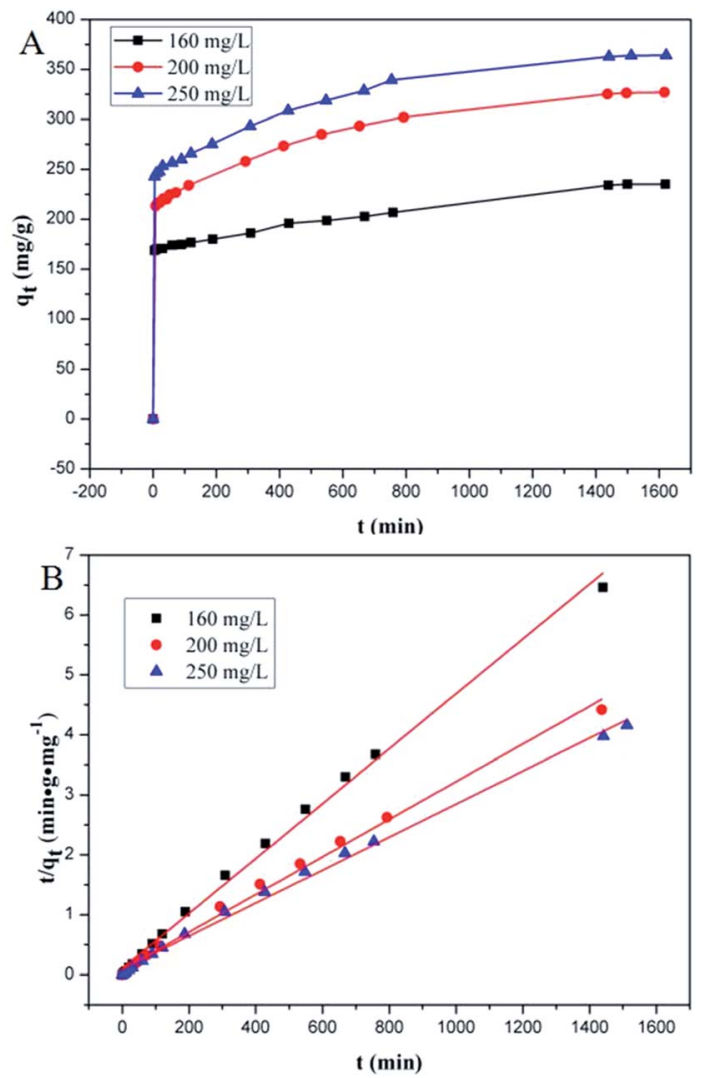

Fig. 2 (A) The adsorption capacity of PEI-PVA microspheres for different concentrations of $\mathrm{Cr}(\mathrm{VI})$ ions with time. (B) The pseudosecond-order model for the adsorption of $\mathrm{Cr}(\mathrm{VI})$ ions by PEI-PVA microspheres. 
where $q_{\mathrm{e}}$ and $q_{t}\left(\mathrm{mg} \mathrm{g}^{-1}\right)$ are the adsorption capacities at equilibrium and at time $t(\mathrm{~min})$, respectively; $K_{1}\left(\mathrm{~min}^{-1}\right)$ and $K_{2}(\mathrm{~g}$ $\mathrm{mg}^{-1} \min ^{-1}$ ) are pseudo-first-order rate constant and pseudosecond-order rate constant, respectively. The effect of the initial concentration $\left(C_{0}\right)$ of $\mathrm{Cr}(\mathrm{vI})$ ions in the aqueous solution on the adsorption capacity of PEI-PVA microspheres is shown in Fig. 2 .

In this study, adsorption kinetics at $25{ }^{\circ} \mathrm{C}$ and $\mathrm{pH}=2$ were obtained for different initial $\mathrm{Cr}$ (vI) concentrations. As shown in Fig. 2A, the adsorption rate was considerably fast at the beginning, but the adsorption equilibrium for different initial concentrations was achieved after about 1 day with no significant difference. The rapid adsorption rate at the beginning implies that there is a high affinity between $\mathrm{Cr}(\mathrm{vI})$ and the PEI-PVA microspheres. Fig. $2 \mathrm{~B}$ and Table $\mathrm{S} 3 \dagger$ show the pseudo-secondorder model for the adsorption of $\mathrm{Cr}(\mathrm{vI})$ by PEI-PVA microspheres.

\subsection{Practical application of PEI-PVA functionalized films}

After the water evaporated, the circumference of the PVA film on the dish was carved out, and the film was peeled off using tweezers; the films obtained were thin, transparent, and mechanically durable. We put a PEI-PVA functional film in a chromium ion solution to test its degradation efficiency in this particular application. When the whole film was put into the $\mathrm{Cr}(\mathrm{vI})$ ion solution, it quickly became soft but was not damaged. This is because PVA has good water solubility and the film has good mechanical properties (Fig. 3). After 20 minutes, it was observed that the solution changed from an initial bright yellow to a pale yellow, and the color gradually became lighter as time passed and eventually became colorless. After 90 minutes, we removed the film from the $\mathrm{Cr}(\mathrm{vI})$ solution and found that the solution was almost colorless, the film morphology was not damaged, and no residual metal was left in the solution; the whole progress is shown in Fig. 3. These results show that the PEI-PVA microspheres contained in the functionalized film do not leak into the solution and have a good ability to remove $\mathrm{Cr}(\mathrm{vI})$ in the actual chromium ion solution treatment. Because no complicated adsorbent separation method is used, the cost will be greatly reduced.

\subsection{Effect of coexisting ions and reusability}

Industrial wastewater containing $\mathrm{Cr}(\mathrm{vI})$ ions also contain a large amount of other heavy metal ions that can compete with $\mathrm{Cr}(\mathrm{vI})$ for adsorption and lower its removal efficiency. ${ }^{37}$ Considering this situation, we have selected some ions to investigate the competitive influence of these co-existing ions on $\mathrm{Cr}(\mathrm{vI})$ removal using our PEI-PVA functional film, and the results are presented in Fig. 4. An initial $100 \% \mathrm{Cr}(\mathrm{vI})$ removal was recorded using $50 \mathrm{~mL}$ of $200 \mathrm{mg} \mathrm{L}^{-1}$ of $\mathrm{Cr}(\mathrm{vI})$ solution ( $\mathrm{pH}$ at 2) and $25 \mathrm{mg}$ of $\mathrm{PPF}$, and the results show that different concentrations of coexisting ions in the solution, both anions and cations, do not significantly affect the removal efficiency of $\mathrm{Cr}(\mathrm{vI})$. The results can be explained by the presence of chromium ions in low $\mathrm{pH}$ solutions and the surface properties of PPF. At low pH, the cations in the solution are repelled from the positively charged surface of the PEI modified PVA surface because PEI modification introduces plenty of $-\mathrm{NH}_{2}$ onto the surface of PVA and

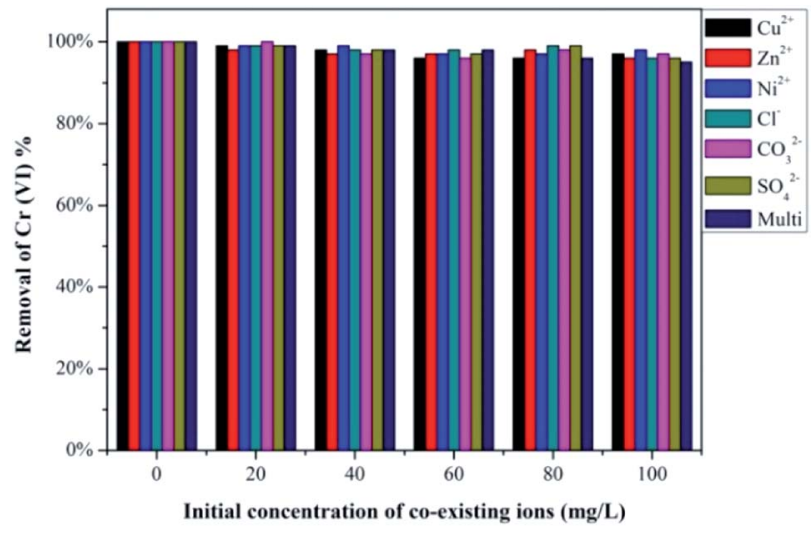

Fig. 4 Effect of co-existing ions on the removal of $\mathrm{Cr}(\mathrm{vI})$ by PPF.

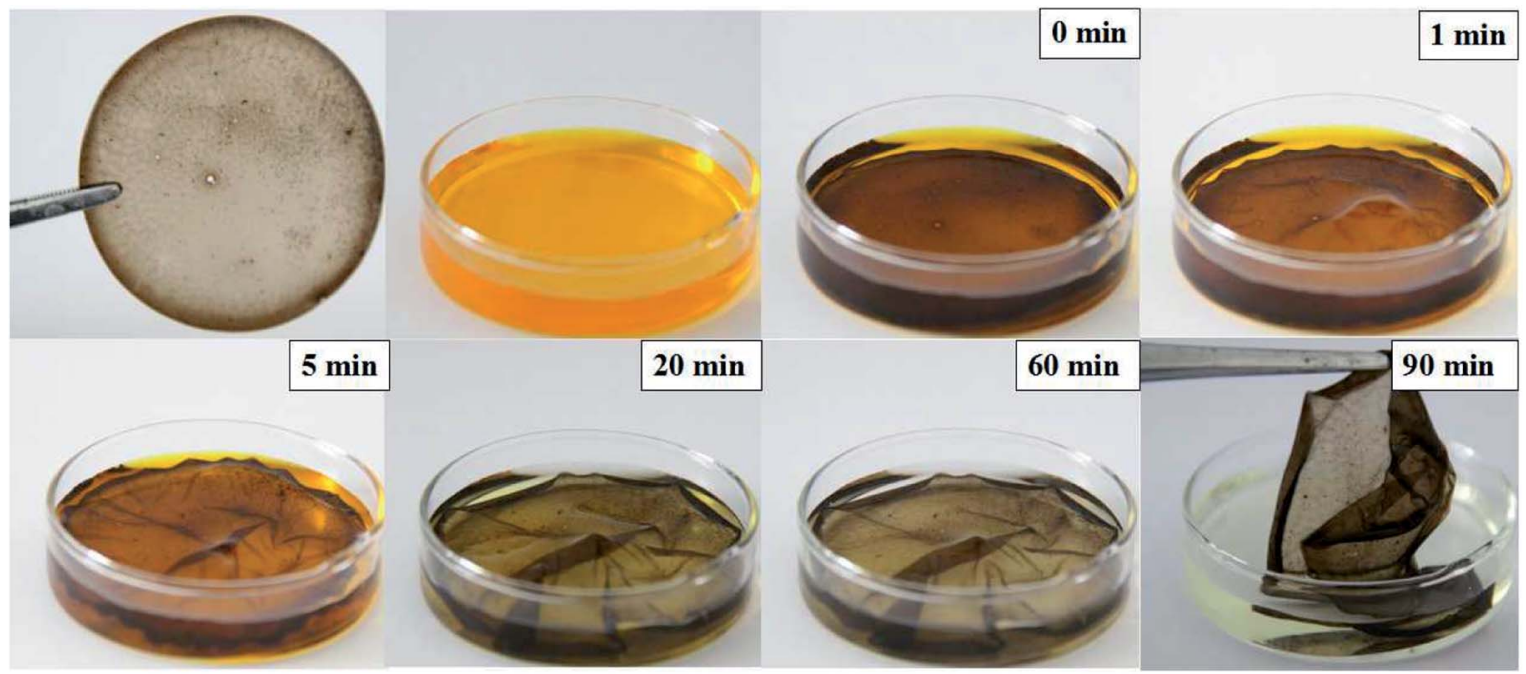

Fig. 3 Photographs of PEI-PVA functionalized films during their practical application in $\mathrm{Cr}(\mathrm{VI})$ ion solutions at different times. 
$-\mathrm{NH}_{2}$ tend to be protonated to $-\mathrm{NH}_{3}{ }^{+}$under acidic conditions, and therefore, the presence of cations do not affect $\mathrm{Cr}(\mathrm{vI})$ removal as expected. Anions such as $\mathrm{Cl}^{-}, \mathrm{CO}_{3}{ }^{2-}$ and $\mathrm{SO}_{4}{ }^{2-}$ are expected to compete with $\mathrm{Cr}(\mathrm{vI})$ for positively charged adsorption sites on the PPF surface because at low $\mathrm{pH}$, chromium ions exist in the solution in the form of $\mathrm{HCrO}_{4}{ }^{-}$. However, this result was not observed as seen in Fig. 4 . This could be due to the weak interaction between the adsorption sites on the PPF and the $\mathrm{Cl}^{-}, \mathrm{CO}_{3}{ }^{2-}$ and $\mathrm{SO}_{4}{ }^{2-}$ anions in the solution. The high selectivity of PPF to $\mathrm{Cr}(\mathrm{vI})$ is also attributed to the reduction of $\mathrm{Cr}(\mathrm{vI})$ to $\mathrm{Cr}$ (III) by the electron-rich PEI polymer, which causes the equilibrium to move forward, thus promoting further adsorption and reduction of $\mathrm{Cr}(\mathrm{vI})$. $\mathrm{As} \mathrm{Cl}^{-}, \mathrm{CO}_{3}{ }^{2-}$ and $\mathrm{SO}_{4}{ }^{2-}$ are weaker oxidizing agents than $\mathrm{HCrO}_{4}{ }^{-}(\mathrm{Cr}(\mathrm{vI}))$, they are not reduced by $\mathrm{PPF}$ and therefore do not affect $\mathrm{Cr}(\mathrm{vI})$ adsorption. ${ }^{38}$

In addition, reusability is also one of the most important considerations in the suitability of adsorbents because better recyclability greatly reduces the overall cost of the adsorbent. In this study, we showed that the adsorption of $\mathrm{Cr}(\mathrm{vI})$ ions onto our PEI-PVA functional film is $\mathrm{pH}$-dependent, and that lower $\mathrm{pH}$ values are suitable for $\mathrm{Cr}(\mathrm{vI})$ adsorption; therefore, the desorption of $\mathrm{Cr}(\mathrm{vI})$ ions from the adsorbent can be achieved by increasing the system's pH. Hence, $\mathrm{Cr}(\mathrm{vI})$-adsorbed PPF was eluted using $0.5 \mathrm{M} \mathrm{NaOH}$ solution for $12 \mathrm{~h}$, and then rinsed with distilled water. To regenerate the adsorbent and desorb any $\mathrm{Cr}$ species, the PPF was then treated with $2 \mathrm{M} \mathrm{HCl}$ and was subjected to a number of recycling cycles as shown in Fig. 5. After 4 cycles, the PPF still possessed more than $77 \%$ removal efficiency for $\mathrm{Cr}(\mathrm{vI})$, indicating that PPFs have good reusability in $\mathrm{Cr}(\mathrm{vI})$ adsorption. The removal efficiency of PPFs for $\mathrm{Cr}(\mathrm{vI})$ decreased by $9.4 \%$ in the third cycle, and this result indicates that PPFs can be easily regenerated and repeatedly utilized in the removal of $\mathrm{Cr}(\mathrm{vI})$ from wastewater.

\subsection{Investigation of the $\mathrm{Cr}(\mathrm{vI})$ removal mechanism}

In order to obtain details on the interaction between $\mathrm{Cr}(\mathrm{vI})$ and PEI-PVA microspheres, XPS spectra of PPF before and after the

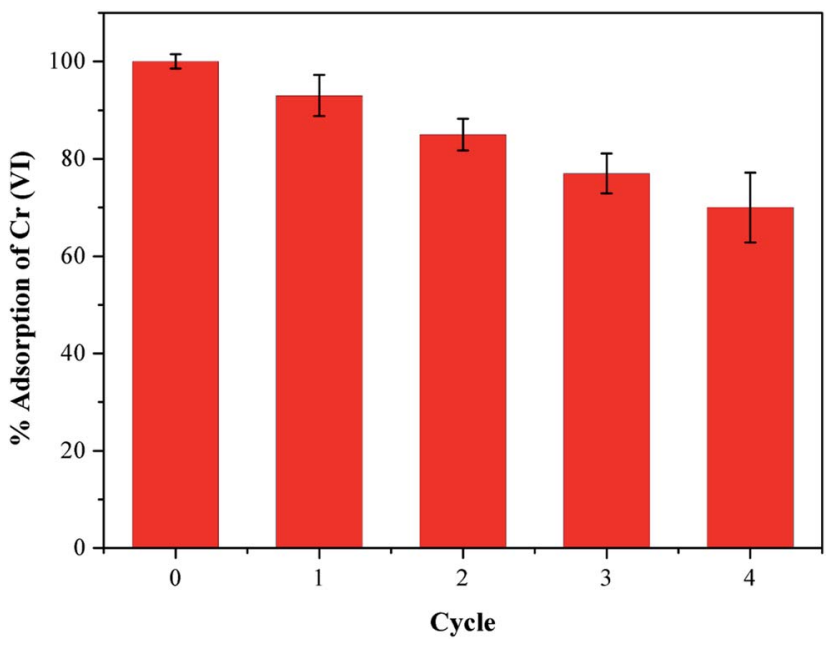

Fig. 5 Reusability of PPF for $\mathrm{Cr}(\mathrm{vI})$ ion adsorption. $\left(\mathrm{pH}=2,25^{\circ} \mathrm{C}\right.$, initial concentration $=200 \mathrm{mg} \mathrm{L}^{-1}$ ). adsorption of $\mathrm{Cr}(\mathrm{vI})$ were obtained and are shown in Fig. S4. $\dagger$ As shown in Fig. S4B, $\uparrow$ the peaks at 284.6 and $286.5 \mathrm{eV}$ of PPF correspond to $\mathrm{C}-\mathrm{C}$ and $\mathrm{C}-\mathrm{O}$ bonds in PVA, respectively. In Fig. S4C, $\dagger$ before the adsorption of $\mathrm{Cr}(\mathrm{vI})$, no $\mathrm{Cr}$ ion signals are observed in the XPS spectra. However, two energy bands at 577.3 and $586.8 \mathrm{eV}$ appear after the adsorption of $\mathrm{Cr}(\mathrm{vI})$, corresponding to the binding energies of $\mathrm{Cr} 2 \mathrm{p}_{3 / 2}$ and $\mathrm{Cr} 2 \mathrm{p}_{1 / 2} \cdot{ }^{39}$ This observation suggests the existence of both $\mathrm{Cr}(\mathrm{III})$ and $\mathrm{Cr}(\mathrm{VI})$ on the surface of PPF after their adsorption of $\mathrm{Cr}(\mathrm{vI})$, indicating that $\mathrm{Cr}(\mathrm{VI})$ was reduced to $\mathrm{Cr}(\mathrm{III})$ during the adsorption process. As shown in Fig. S4D, $\dagger$ after the treatment of $\mathrm{Cr}(\mathrm{VI})$, the $\mathrm{N} 1 \mathrm{~s}$ peak intensity of PPF decreased significantly, indicating that $-\mathrm{NH}_{2}$ of PPF is involved in the treatment of $\mathrm{Cr}(\mathrm{vI})$.

On the basis of the above analyses, the mechanism of the removal of $\mathrm{Cr}(\mathrm{VI})$ can be concluded (Scheme $\mathrm{S} 2 \dagger$ ). After a series of chemical reactions, PVA microspheres were combined with PEI, and PEI introduced plenty of $-\mathrm{NH}_{2}$ groups on the PVA microspheres; these amino groups showed a great effect in the application for $\mathrm{Cr}(\mathrm{vI})$ removal. Branched polyethylenimine (PEI) has plenty of primary, secondary and tertiary amines on the macromolecular chains, which are considered as effective functional reagents to modify the adsorbent. ${ }^{34}$ When PPF is in contact with chromium ions in an aqueous solution, it can effectively adsorb $\mathrm{Cr}(\mathrm{vI})$ ions mainly through electrostatic attraction between $-\mathrm{NH}_{3}{ }^{+}$and $\mathrm{Cr}(\mathrm{vI})$ under acidic conditions. Then, the reduction reactions may proceed. The electrons required for the reduction of $\mathrm{Cr}(\mathrm{vI})$ come from the electrondonating groups of the branched polyethylenimine (PEI). Finally, with the help of electrons, $\mathrm{Cr}$ (VI) can be reduced to $\mathrm{Cr}$ (III) and attached to the surface of the PPF by electrostatic attraction.

\section{Conclusions}

In this study, micron-sized polyethylenimine-functionalized poly(vinyl alcohol) microspheres were prepared through a series of chemical reactions. Once generated, the resulting PEI-PVA microspheres were tested for their ability to remove $\mathrm{Cr}(\mathrm{vI})$ from aqueous solutions in batch experiments. The results show that the adsorption of $\mathrm{Cr}(\mathrm{vI})$ is significantly $\mathrm{pH}$ dependent, and the adsorption capacity decreases with the increase in the $\mathrm{pH}$. The optimized $\mathrm{pH}$ value is 2.0. The adsorption isotherm of the adsorbent fits the Langmuir model, with a maximum adsorption capacity of $396.83 \mathrm{mg} \mathrm{g}^{-1}$. The adsorption rate of $\mathrm{Cr}(\mathrm{vI})$ increases rapidly in tens of minutes and gradually reaches the adsorption equilibrium with increasing time. In order to assess the capability of our PEI-functionalized PVA microspheres for $\operatorname{Cr}(\mathrm{vI})$ removal in actual wastewater, we filled the microspheres into a polyvinyl alcohol film to prepare a PEI-PVA functionalized film. We put the film in a chromium ion solution and recorded changes in chromium ion concentration over time. As the results show, the PPF has an obvious effect on the removal of chromium ions. After treatment, the film and solution can be easily separated, and can be effectively reused. The adsorption effect is mainly due to the electrostatic interactions between $\mathrm{Cr}(\mathrm{vI})$ anions in the solution at $\mathrm{pH} 2$ and the protonated amino groups $\left(-\mathrm{NH}_{3}{ }^{+}\right)$present on the film surface. After that, 
some of the adsorbed $\mathrm{Cr}(\mathrm{VI})$ can be reduced to $\mathrm{Cr}$ (III) by the electron-donor groups of branched polyethylenimine (PEI). In addition, the presence of various cations and anions in wastewater has no significant effect on the adsorption of $\mathrm{Cr}(\mathrm{vI})$. We think that this work provides a promising approach for the removal of $\mathrm{Cr}(\mathrm{vI})$ from aqueous solutions and may have a huge application prospect.

\section{Conflicts of interest}

There are no conflicts to declare.

\section{Acknowledgements}

This work was supported by National Natural Science Foundation of China (No. 21605144, 21876175) and Key Research and Development Program of Anhui Province (1804a07020103).

\section{Notes and references}

1 M. K. Dinker and P. S. Kulkarni, J. Chem. Eng. Data, 2015, 60, 2521-2540.

2 J. T. Liu, J. Mater. Chem. A, 2016, 4, 1970-1979.

3 J. Hu, J. Hazard. Mater., 2009, 162, 1542-1550.

4 L. L. Fan, J. Mater. Chem., 2012, 24577-24583.

5 P. F. Zong, Chem. Eng. J., 2013, 220, 45-52.

6 A. Alejandra Pérez-Fonseca, Ind. Eng. Chem. Res., 2011, 59395946.

7 A. Potsangbam, C. Saswati and R. Manabendra, Chem. Eng. J., 2008, 130-140.

8 K. Anupam, Chem. Eng. J., 2011, 135-143.

9 Q. Fan, Catal. Commun., 2013, 42, 14-19.

10 N. Ballav, M. Arjun and S. B. Mishra, Chem. Eng. J., 2012, 198, 536-546.
11 M. Bhaumik, M. Rob and M. Arjun, Chem. Eng. J., 2013, 228, 506-515.

12 N. Ballav, Appl. Clay Sci., 2014, 102, 60-70.

13 Y. Lei, Ind. Eng. Chem. Res., 2012, 31, 10408-10415.

14 J. Q. Wang, J. Hazard. Mater., 2013, 244, 121-129.

15 Z. K. Setshedi, Chem. Eng. J., 2013, 222, 186-197.

16 Y. Chen, $R S C$ Adv., 2014, 34, 17805-17811.

17 M. R. Huang, H. J. Lu and X. G. Li, J. Mater. Chem., 2012, 34, 17685-17699.

18 G. F. Li, J. Colloid Interface Sci., 2013, 409, 43-51.

19 Y. Q. Zhan, Chem. Eng. J., 2018, 333, 132-145.

20 N. Ali, S. Seraj and B. Mirzayi, Chem. Eng. J., 2015, 277, 21-29.

21 D. Barrera, E. Carlos, L. L. Violeta and B. Bryan, J. Hazard. Mater., 2012, 223, 1-12.

22 Y. D. Zou, Environ. Sci. Technol., 2016, 14, 7290-7304.

23 H. L. Ma, J. Mater. Chem., 2012, 13, 5914-5916.

24 Z. Huang, J. Hazard. Mater., 2016, 302, 323-331.

25 X. J. Liu, Chem. Eng. J., 2012, 183, 238-243.

26 H. Wang, J. Hazard. Mater., 2015, 286, 187-194.

27 L. L. Fan, J. Mater. Chem., 2012, 47, 24577-24583.

28 Y. Liu, RSC Adv., 2015, 67, 54156-54164.

29 M. Tuzen and S. Mustafa, J. Hazard. Mater., 2007, 147, 219225.

30 C. Luo, Chem. Eng. J., 2013, 234, 256-265.

31 X. Guo, J. Phys. Chem. C, 2011, 115, 1608-1613.

32 M. Bhaumik, J. Hazard. Mater., 2011, 190, 381-390.

33 M. Bhaumik, Chem. Eng. J., 2012, 181, 323-333.

34 K. R. Zhu, ACS Sustainable Chem. Eng., 2016, 4361-4369.

35 M. Bhaumik, J. Hazard. Mater., 2011, 190, 381-390.

36 D. F. Wang, Langmuir, 2017, 33, 7007-7014.

37 M. Bhaumik, J. Colloid Interface Sci., 2016, 470, 257-267.

38 N. H. Kera, J. Colloid Interface Sci., 2016, 476, 144-157.

39 S. K. Li, J. Colloid Interface Sci., 2012, 378, 30-35. 